\title{
PREDICTION AND MEASUREMENT OF HEAT TRANSFER IN AIR-COOLED DISC-TYPE ELECTRICAL MACHINES
}

\author{
David A. Howey*, Andrew S. Holmes ${ }^{\dagger}$, Keith R. Pullen ${ }^{\ddagger}$ \\ *Department of Mechanical Engineering, Imperial College, Exhibition Road, London, SW7 2AZ, UK \\ Tel. +44 (0)20 7594 1618, Fax. +44 (0)20 7823 8845, Email: d.howey@imperial.ac.uk \\ ${ }^{\dagger}$ Department of Electrical and Electronic Engineering, Imperial College, Exhibition Road, London, SW7 2AZ, UK \\ ${ }^{\ddagger}$ School of Engineering and Mathematical Sciences, Tait Building, City University \\ Northampton Square, London, EC1V 0HB, UK
}

\begin{abstract}
Index Terms-Axial flux permanent magnet machine, disc type machine, stator heat transfer, thermal analysis, CFD

Abstract-Accurate thermal analysis of axial flux permanent magnet (AFPM) machines is crucial in predicting maximum power output. Stator convective heat transfer is one of the most important and least investigated heat transfer mechanisms and is the focus of this paper. Experimental measurements were undertaken using a thin-film electrical heating method, providing radially resolved steady state heat transfer data from an experimental rotor-stator system designed as a geometric mockup of a through-flow ventilated AFPM machine. The measurements are compared with computational fluid dynamics (CFD) simulations using both 2D axisymmetric and 3D models. These were found to give a conservative estimate of heat transfer, with inaccuracies near the edge and in the transitional flow regime. Predicted stator heat transfer was found to be relatively insensitive to the choice of turbulence model and the SST model was used for most of the simulations.
\end{abstract}

\section{INTRODUCTION}

Disc type electrical machines such as the axial flux permanent magnet (AFPM) machine offer high torque density and high efficiency in a compact package and are of great interest for applications such as electric and hybrid vehicles and portable generator sets [1]. Their electromagnetic design is well understood, but far less research has been undertaken on thermal aspects. This is despite the fact that power density is usually limited by maximum temperature. Since the stator temperature that is achieved depends on the amount of specific internal heat generation and the convection heat transfer from the stator surface, an understanding of the latter is essential in machine design.

Significant research, e.g. [2] has been undertaken on the thermal modelling of radial flux (cylindrical) electrical machines. However, the air flow within a radial flux geometry is completely different to the air flow within a disc type machine, and the convective heat transfer results which are relevant to the former case cannot be applied to the latter. Recent work on the thermal aspects of disc type machines [3]-[5] has tended to focus on one-dimensional lumped parameter (LP) networks to represent both the solid domain and the fluid domain. These are fast to solve and widely used in electrical machine design. However, the LP method is only as good as the input correlations used for thermal contact resistances, convective heat transfer coefficients and air flow modelling and therefore CFD and finite element analysis (FEA) are being increasingly applied to investigate the thermal design of electrical machines in more detail, combined with experimental validation.

AFPM machines are inherently more three dimensional in nature than radial flux machines and the air flow in the rotorstator gap sees an expanding cross sectional area from inside to outside, unlike in a radial flux geometry. All authors comment that suitable stator convective heat transfer coefficients could not be found in the literature, and therefore they either apply rotor correlations (which are likely to lead to an overoptimistic assessment of heat transfer), or use CFD simulations to predict heat transfer coefficients. Few studies have applied CFD to AFPM machines. Airoldi et al. [6], [7] undertook CFD modelling of the fluid flow in an AFPM machine, finding good correspondence between CFD and experiment for mass flow rates and temperatures, although a limited experimental data set is presented.

We have recently reported direct experimental measurements of stator heat transfer in a rotor-stator system using a flat rotor and flat stator as an idealized geometry [8], [9]. Heat transfer in the form of non-dimensional Nusselt number was measured at stator radii $0.6<r / R<1$, at three gap ratios relevant to disc type electrical machines. Transition from laminar to turbulent flow was observed to begin at rotational Reynolds numbers $R e_{\theta}>3 e 5$. It was also shown that the ingress of ambient air at the periphery of the stator has a significant effect on stator heat transfer.

In this paper, measurements are compared with CFD simulations using both 2D axisymmetric and 3D models. Results have been non-dimensionalised using various dimensionless groups: rotational Reynolds number $R e_{\theta}$, Nusselt number $N u$, gap ratio $G$ and non-dimensional air mass flow rate $C_{w}$. These are defined as follows:

$$
\begin{gathered}
R e_{\theta}=\frac{\omega R^{2}}{\nu} \\
N u=\frac{h R}{k}, \overline{N u}=\frac{\bar{h} R}{k} \\
G=\frac{g}{R} \\
C_{w}=\frac{\dot{m}}{\mu R}
\end{gathered}
$$


where $R$ is the rotor outside radius, $\omega$ is the rotor speed, $\nu$ is the fluid kinematic viscosity at ambient temperature, $h$ is the convective heat transfer coefficient, $k$ is the fluid conductivity at ambient temperature, $g$ is the gap size between rotor and stator, $\dot{m}$ is the air mass flow rate and $\mu$ is the dynamic viscosity. Also, radial position is non-dimensionalised to $r / R$.

The flow between a rotating and a stationary disk can be divided into: Batchelor flow, where there is a rotating core of fluid between separate laminar or turbulent boundary layers; and Stewartson flow, where the tangential velocity drops away from the rotor surface to nearly zero at the stator surface, with no core rotation. The condition at the periphery determines the flow type. Batchelor flow is observed in enclosed systems where a recirculation of fluid from rotor to stator occurs at the periphery. In systems which are open at the periphery and have radial through-flow, Stewartson flow is seen. There is a smooth transition from one flow type to the other, as discussed by Poncet et al. [10], depending on the amount of radial through-flow. This may vary from one type of AFPM machine to another.

Stator heat transfer has been investigated experimentally by Owen et al. [11], Bunker et al. [12] and Yuan et al. [13], as well as in CFD by Iacovides and Chew [14]. However to date only a very limited range of parameters have been investigated. Generalised correlations that might be applied in the design of AFPM machines are not available in the literature.

\section{EXPERIMENTAL RIG}

Given the wide variation in available geometries of AFPM machines, it was decided in the present work to focus mainly on a simplified through-flow ventilated rotor-stator system with a flat rotor and a flat stator. Real electrical machines are more complex than this, but studying this simpler system allows comparison with historical work on rotor-stator systems and provides a basic understanding of stator convective heat transfer, as well as tending to give a conservative estimate of stator heat transfer. A brief exploration of the effect of rotor protrusions (such as permanent magnets) on the air flow has also been undertaken.

An experimental rig was constructed to measure stator heat transfer, consisting of a $471 \mathrm{~mm}$ diameter rotor driven by a servo motor, and a heated stator surface allowing direct measurement of stator heat transfer, with 14 channels of spatial resolution and a measurement accuracy of about $\pm 8 \%$. The measurement technique used a thin-film electrical heating method and is fully described in [8], [9]. Two rotor configurations were tested: a flat rotor (R1), and a rotor with 16 protrusions designed to mimic the magnets on an AFPM machine (R2), shown in figure 1 . The protrusions act like crude fan blades. The gap ratio $G$ was adjustable from 0.01 to 0.09 by insertion of accurately machined spacers. Air was pumped by the movement of the rotor from an inlet at the stator centre to four exits at the edge of the rotor-stator system. The air mass flow rate was measured using a calibrated bellmouth entry.

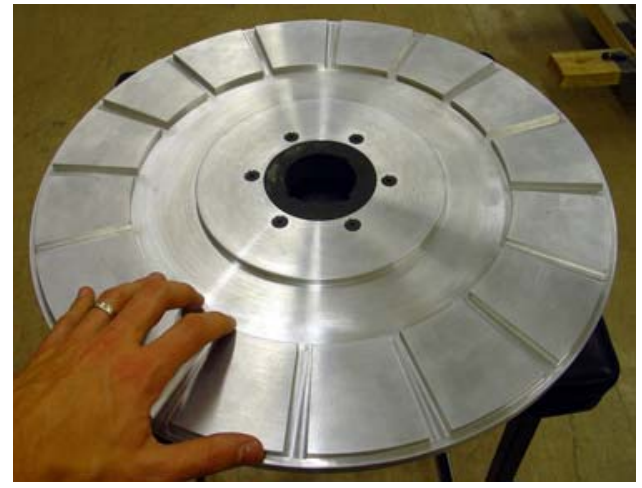

Fig. 1. Photograph of R2, aluminium rotor with protrusions

\section{CFD MODEL}

Computational fluid mechanics is concerned with the numerical solution of the equations governing fluid flow. The commercial software Ansys CFX was used in the present study. Rotor-stator flows exhibit a number of phenomena which are challenging for CFD, such as rotation, confinement, heat transfer, separation, transition and turbulence.

It has been found [15] that 2D axisymmetric CFD simulations are able to capture the important characteristics of rotor-stator flow. Since 2D models are substantially faster to solve, but give useful results, their use in the present work allowed a number of flow speeds and gap sizes to be investigated. Subsequent $3 \mathrm{D}$ modelling was then informed by the $2 \mathrm{D}$ results but only one gap size was investigated. In order to predict the mass flow rate correctly, modelling of the air inlet geometry could not be neglected, and this was only possible using the $2 \mathrm{D}$ model. Because the inlet geometry was not modelled in $3 \mathrm{D}$, the inlet total pressure boundary condition for the $3 \mathrm{D}$ model was derived from the $2 \mathrm{D}$ models.

Table I gives a brief summary of the various CFD simulations that were undertaken.

TABLE I

CFD SCENARIOS

\begin{tabular}{lcc}
\hline \hline & 2D simulations & 3D simulations \\
\hline Gap ratio & $G=0.0106,0.0212$ & $G=0.0106$ \\
Outer edge & open & open, partially blocked \\
Turbulence & laminar, turbulent & laminar, transitional, turbulent \\
Rotor & R1 only & R1 and R2 \\
\hline
\end{tabular}

The Shear Stress Transport (SST) turbulence model was chosen for modelling turbulent flow. This is an improved twoequation model available in CFX, developed by Menter [16]. It is a combination of the $k-\omega$ and $k-\epsilon$ models. Near a wall, twoequation turbulence models need to be modified to account for molecular viscosity $\mu$. This is achieved either by using a wall function, which does not require a fine mesh by the 
wall, or by using a fine mesh and adding additional terms in the near wall region and integrating directly through the viscous sub-layer. The CFX software includes algorithms that automatically select the latter formulation near the wall if the grid is fine enough. This requires $y^{+} \approx 1\left(y^{+}\right.$is the nondimensional distance from the wall to the first grid point, it depends on the flow solution). In the present work, modelling was undertaken wherever possible with $y^{+}<1$ to avoid the use of wall functions, although in the case of rotor R2 simulations, this was not possible.

Whether the flow is laminar or turbulent has a large influence on the wall heat transfer and therefore assumptions must be made about transition. In the case of the free rotor, transition is known to begin at $1.8 e 5<R e_{\theta}<2.1 e 5$ and completely turbulent flow is found for $R e_{\theta}>3 e 5$ [17]. It was decided initially to assume the flow was completely laminar for simulations below $600 \mathrm{rpm}$ and fully turbulent for speeds of 1800 rpm and above. Between these speeds, both fully turbulent SST modelling and transition modelling were attempted using the Langtry and Menter $\gamma-\theta$ transition model [18]. Transition modelling for the 3D model of R1 was attempted using the $\gamma-\theta$ model with default settings.

In all simulations it was assumed that air was an ideal gas with constant viscosity, thermal conductivity and specific heat capacity. At the temperatures and speeds of interest these properties do not vary considerably and do not have a large affect on the parameters of interest.

Figure 2 shows the simulated 2D fluid domain, superimposed on a drawing of the experimental rig. The air inlet geometry was modelled excluding the bellmouth itself (which is expected to have low losses).

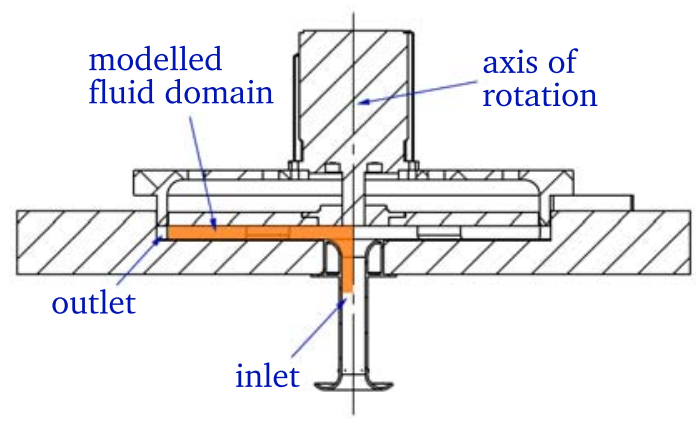

Fig. 2. Fluid domain used for 2D CFD simulation (shaded), relative to rig

One quarter of the entire rotor-stator domain was modelled in $3 \mathrm{D}$ for rotor $\mathrm{R} 1$, since there are four air exits in the experimental rig. The inlet geometry was not modelled, instead the domain begins at a radius of $38.1 \mathrm{~mm}$; this radius was chosen since it is the point where the curved inlet from the bellmouth and pipe comes to an end. Figure 3 shows the 3D modelled fluid domain in the context of the experimental rig.

In addition to the simplified geometry with a flat rotor (R1), a brief CFD study was also carried out on a rotor with

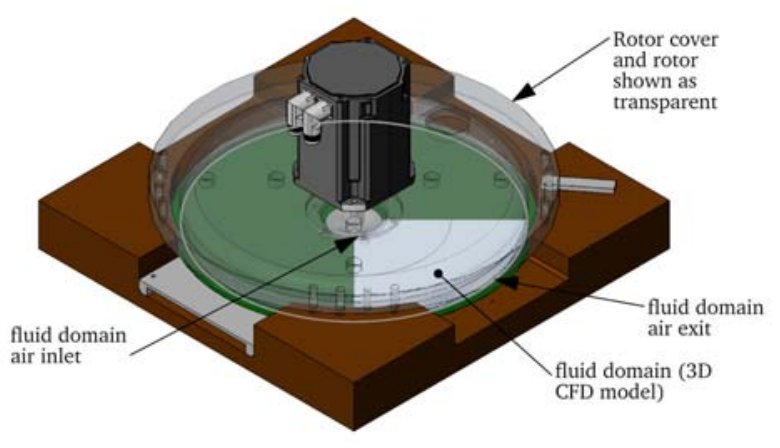

Fig. 3. 3D Fluid domain geometry in context

protrusions (R2), mimicking the permanent magnets of an AFPM machine with gap ratio 0.0106 .

CFX-mesh was used for the meshing of the 2D geometries. There were approximately 40 elements across the gap in the $z$ direction and approximately 100,000 elements in total. Meshing for the 3D geometry with rotor R1 was accomplished using Ansys ICEM to produce a fan-shaped regular hexahedral mesh with inflated layers on the rotor and stator with approximately 500,000 elements. Meshing for R2 was accomplished using Ansys v12 meshing software to produce an irregular mesh with inflated boundary layers with approximately 425,000 elements. In all R1 simulations, $y^{+}<1$ was achieved. In R2 simulations, the largest value of $y^{+}$was approximately 14 . Further mesh refinement for $\mathrm{R} 2$ in an attempt to achieve $y^{+} \approx 1$ led to failure due to computer memory limitations.

The boundary conditions for rotor R1 (flat) are given in table II. In the case of the $3 \mathrm{D}$ simulations, the inlet total pressure was decreased to a negative value to reflect losses in the inlet. In the simulations using rotor $\mathrm{R} 2$, the inlet mass flow rate was specified directly from experimental results, instead of specifying inlet total pressure. Additionally, R2 simulations used a rotating reference frame for the fluid domain, with boundary conditions specified in relation to this.

TABLE II

BOUNDARY CONDITIONS USED FOR R1 SIMULATIONS

\begin{tabular}{ll}
\hline \hline Boundary & Condition \\
\hline Inlet & $p_{\text {total }}=0 \mathrm{~Pa}, T=293 \mathrm{~K}$ \\
Outlet & $p_{\text {static }}=0 \mathrm{~Pa}$ \\
Rotor & No-slip rotating wall \\
Stator & No-slip stationary adiabatic wall \\
Heater & No-slip stationary isothermal wall $(333 \mathrm{~K})$ \\
\hline
\end{tabular}

Grid independence tests for were undertaken at $R e_{\theta}=9.6 e 5$ to investigate the effect of grid size on $N u(r)$, and grid independence at both gap sizes was achieved.

Solver controls were specified as (1) a convergence criterion and (2) a domain balance criterion. The convergence criterion was that the maximum residual should be less than 1e-4. A domain imbalance (of momentum and enthalpy) of less than $1 \%$ was also specified as a requirement. In general, $2 \mathrm{D}$ and $3 \mathrm{D}$ 
simulations converged well to these conditions in both laminar and turbulent cases, with the maximum residuals usually less than 1e-5 and sometimes approaching 1e-6.

\section{EXPERIMENTAL RESULTS}

Figure 4 shows measured average stator heat transfer versus rotational Reynolds number for various gap sizes using R1 (flat rotor). Higher speed tests were conducted at two gap sizes $G=0.0106,0.0212$ as shown.
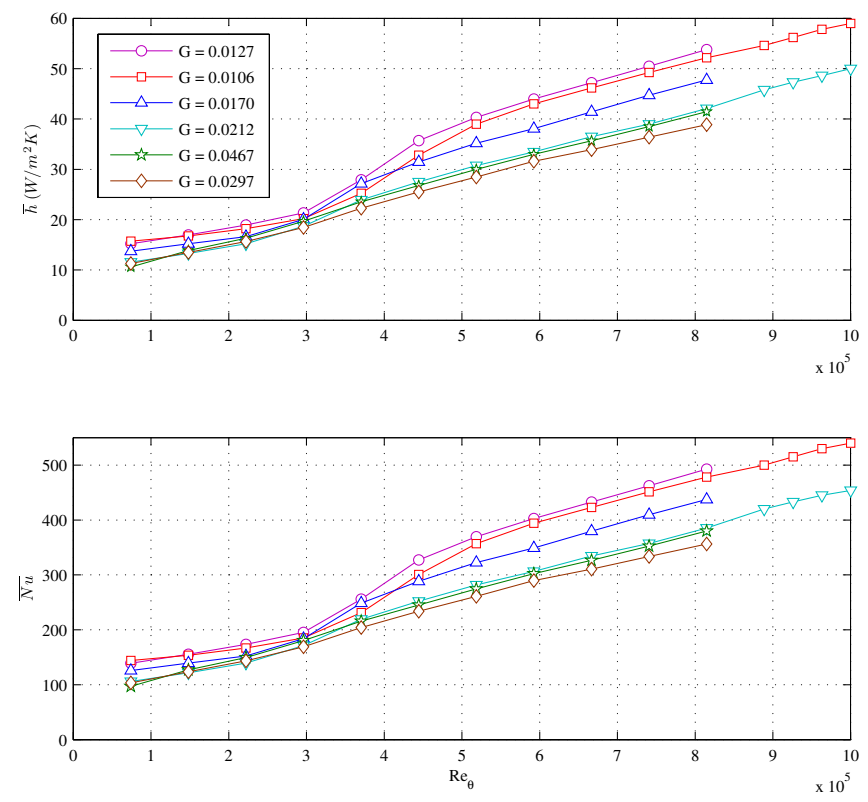

Fig. 4. Average heat transfer coefficients and Nusselt numbers, rotor R1

The average heat transfer results can be correlated in the turbulent regime according to:

$$
\overline{N u}_{t u r b}=A R e_{\theta}^{B}
$$

The constants $A$ and $B$ are given in table III; these are valid in the range $R e_{\theta} \geq 5.19 e 5$ for the data shown. All values of $B$ are similar, $B=0.673 \pm 0.028$. Values of $A$ differ according to gap size $G$.

TABLE III

AVERAGE HEAT TRANSFER TURBULENT CORRELATIONS

\begin{tabular}{ccc}
\hline \hline $\mathrm{G}$ & $\mathrm{A}$ & $\mathrm{B}$ \\
\hline 0.0106 & 0.0790 & 0.640 \\
0.0127 & 0.0888 & 0.633 \\
0.0170 & 0.0406 & 0.682 \\
0.0212 & 0.0315 & 0.691 \\
0.0297 & 0.0347 & 0.679 \\
0.0467 & 0.0234 & 0.712
\end{tabular}

In the laminar regime $\left(R e_{\theta} \leq 3 e 5\right)$, the correlation is expected to have the same functional form i.e. $\overline{N u}_{l a m}=A R e_{\theta}^{B}$. However, there were a limited number of measured data points in this regime (only four per gap ratio) and additionally the situation is complicated by the non-adiabatic rotor condition (discussed below). Nonetheless the results are approximately correlated according to table IV.

TABLE IV

AVERAGE HEAT TRANSFER LAMINAR CORRELATIONS

\begin{tabular}{ccc}
\hline \hline $\mathrm{G}$ & $\mathrm{A}$ & $\mathrm{B}$ \\
\hline $0.0106-0.0127$ & 14.02 & 0.204 \\
0.0170 & 7.249 & 0.252 \\
$0.0212-0.0297$ & 2.359 & 0.336 \\
0.0467 & 0.739 & 0.434 \\
\hline
\end{tabular}

\section{COMPARISON AND DISCUSSION}

\section{A. Flat rotor $(R 1)$}

CFD simulations using the R1 geometry showed a Stewartson flow profile in the laminar regime, but a Batchelor profile with core rotation in the fully turbulent regime. At low speeds an inbound flow recirculation at the stator was observed in all simulated configurations, but at higher speeds this is only prevalent at $G>0.0212$. The simulated temperature profiles in general show the fluid temperature increasing with radius as the fluid is pumped across the heated region. The turbulent thermal boundary layers are much thinner than the laminar layers. The influence of ambient temperature air at the periphery can be seen at both gap ratios, particularly near the edge where there is a drop in fluid temperature near the stator. This is much more prevalent at the larger gap ratio, in both laminar and turbulent results. Some features of the velocity profiles are illustrated in figure 5. In general, there is a distinct difference between the laminar and turbulent regimes, but the non-dimensionalised turbulent profiles are all quite similar across different speeds.
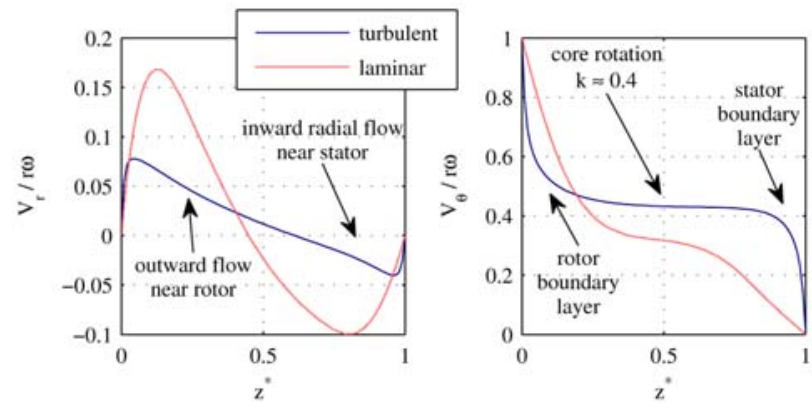

Fig. 5. Velocity profiles, $r / R=0.99, G=0.0212$

Radially resolved heat transfer simulations and measurements for R1 exhibited certain common features for both gap sizes in the 2D and 3D cases. Firstly, Nusselt number increases with increasing $R e_{\theta}$. At the outer radii the Nusselt number increases due to ingress of ambient temperature air. Average measured heat transfer decreases with increased gap size, although there is a small increase from $G=0.0106$ to $G=0.0127$ due to the slightly greater ingress of ambient temperature air at the periphery. Transition from laminar to turbulent flow is 
observed at $R e_{\theta}>3 e 5$, but this is only pronounced at gap ratios $G<0.02$ as shown in figure 4 .

The correspondence between radially resolved heat transfer measurements vs. CFD is good at low speeds (laminar) and $G=0.0212$. At gap ratio $G=0.0106$ low speed correspondence is less good and most of the experimental heat transfer results are higher than the CFD predictions by approximately $50 \%$. This is probably because the CFD boundary condition assumption of an adiabatic rotor becomes invalid at low rotational speeds and small gap sizes, where in experiments there is significant heat transfer into the aluminium rotor resulting in higher measured heat transfer.

It was found that the fully turbulent regime CFD simulations tended to always over-predict the air mass flow rate through the system compared with experimental measurements. This is probably because the total pressure loss from ambient conditions through the bellmouth and entry into the rotor-stator gap in the experimental rig is higher than predicted using CFD. As a result of this, a number of fully turbulent CFD simulations were re-run using the experimentally measured mass flow rates as inlet boundary conditions so that heat transfer could be compared in a like-for-like manner. Figure 6 shows the resulting comparison. As can be seen, the simulated results are now further from the measurements. The reason for the discrepancy is due to edge effects as will now be discussed.

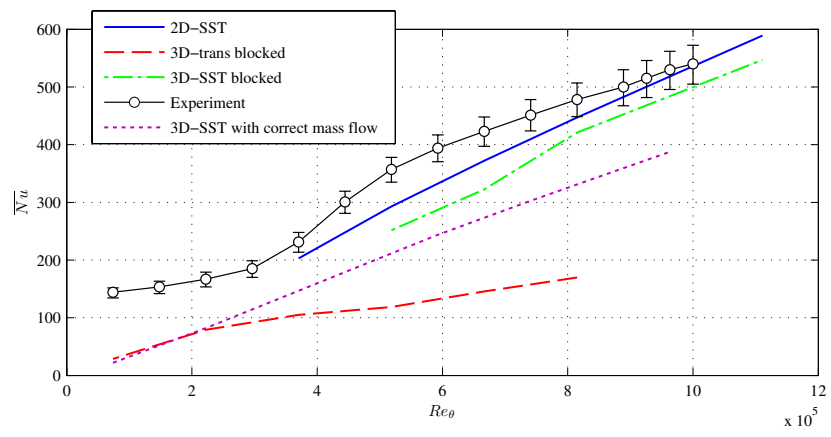

Fig. 6. $G=0.0106$ comparison CFD and experiment

Figure 7 shows a comparison of the radially resolved predictions at $G=0.0106$ and $R e_{\theta}=9.6 e 5$ with the experimentally determined inlet mass flow, using the baseline Reynolds stress model (BSLRSM), SST model with and without curvature correction (SST curv. corr.) and $k-\epsilon$ model, in both 2D and 3D (blocked periphery) cases. As can be seen, choice of turbulence model does not have a significant influence on heat transfer predictions. In the region $0.6<r / R<0.85$ the match between CFD and experiment is quite close. In the region $0.85<r / R<1$ all results deviate substantially from the experimental measurements and this is the cause of the deviation seen in the average results. It is generally accepted that modelling of edge effects is difficult; this deviation is probably caused by the differences in geometry at the boundary in the CFD model compared with the experimental rig.

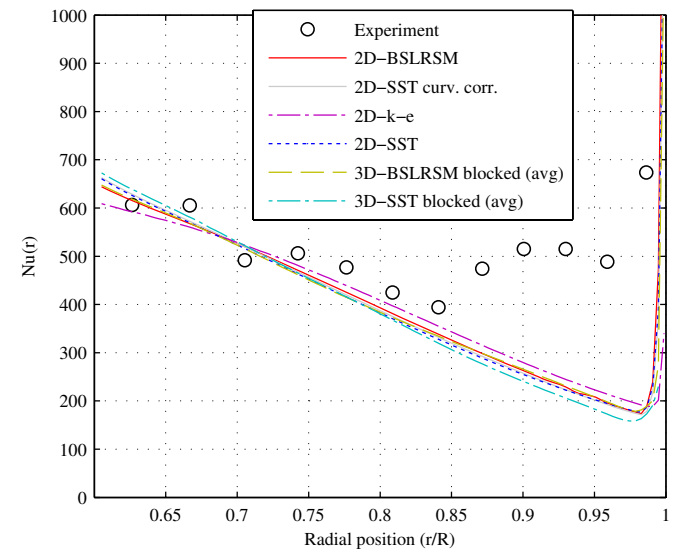

Fig. 7. $G=0.0106$ comparison CFD \& experiment at $R e_{\theta}=9.6 e 5$

The radially resolved comparison results for $G=0.0212$ at this speed showed a closer correspondence to experiment than the smaller gap ratio particularly in the region $0.85<r / R<$ 1. In the transitional regime, the correspondence between CFD and experiment is not as good as in the fully turbulent regime. Transition modelling does not conclusively provide a better fit.

\section{B. Rotor with protrusions (R2)}

The CFD simulated non-dimensional velocity and temperature profiles in the gap for rotor R2 were found to be similar in some respects to the profiles obtained for R1. For example, the turbulent tangential velocity profiles exhibited core rotation in the gap, but the laminar profiles showed no core rotation. However, the radial velocity profiles showed a very different flow pattern to $\mathrm{R} 1$. It was found that the main route for radial outflow of fluid was in the slot between protrusions and only a small amount of fluid flows out near the rotor underneath the protrusions; this agrees well with the simulation results of Airoldi et al. [7]. The streamlines, figure 8, show that fluid is entrained into the channel between magnets/ protrusions and flung outwards, like a crude radial fan.

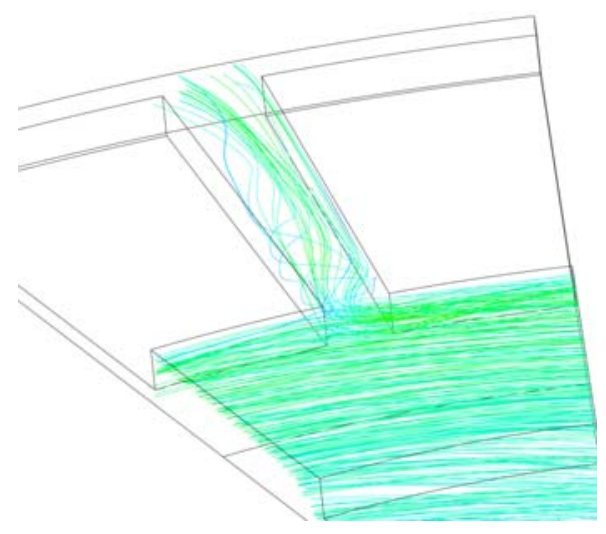

Fig. 8. Streamlines of rotor R2 in the rotating reference frame

The experimentally measured results for $\mathrm{R} 2$ show that the gap 
ratio seems to have much less effect on the heat transfer than for R1. Additionally, the clear regime change from laminar to turbulent flow at $R e_{\theta}>3 e 5$ that occurs with $\mathrm{R} 1$ cannot be seen for R2; transition probably occurs at a much lower rotational speed.

A comparison between measured results for R1 and R2 shows that at a comparable speed, average heat transfer with $\mathrm{R} 2$ is about $20-30 \%$ higher than $\mathrm{R} 1$. This result is similar to the CFD comparison but slightly less marked, figure 9.

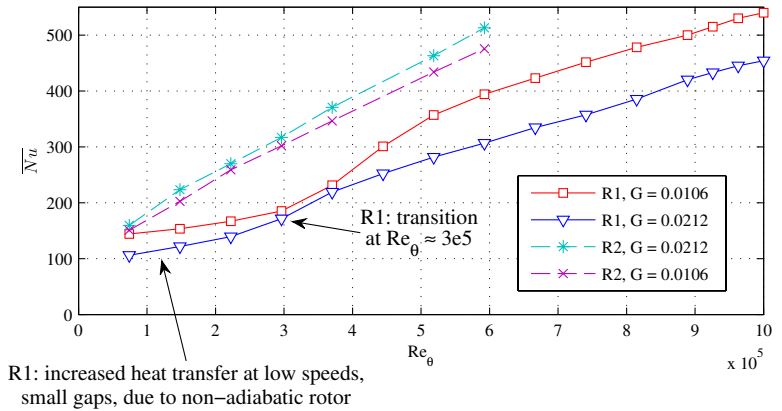

Fig. 9. Comparison of measured average heat transfer R1 vs. R2

Comparison of radially resolved simulated and measured heat transfer for rotor R2 show significant differences at all radial locations. The most likely cause is that the assumption of an open periphery in the case of R2 is completely invalid; in the experimental rig the fluid in fact pulses through the radial slots in $\mathrm{R} 2$ as the rotor moves past consecutive open and closed sections of the periphery. This could be heard during testing as an audible hum at sixteen times the rotor frequency. In order to resolve this more accurately in CFD, a transient simulation with a moving mesh would be required, since there is interference between the slots in R2 and the open and blocked areas of the periphery in much the same way as the nozzle guide vanes and rotor blades interact in a turbine. This was outside the scope of the present work but is a worthwhile area of future work.

\section{CONCLUSIONS}

This work has compared CFD simulations with experimental measurements of stator heat transfer in a rotor-stator disc system, with two rotor types. It was found in general that stator heat transfer is always lower than rotor heat transfer. It was also found that CFD modelling is relatively insensitive to the choice of turbulence model for this problem, but accuracy in modelling boundary conditions is very important. There is some comfort to be gained in the result that the CFD is generally conservative. Investigation of the effects of outlet geometry and magnet geometries in the form of rotor protrusions is an area where further research is needed. Correlations have been given for average stator heat transfer in a range of conditions; these should be useful to AFPM machine designers in providing conservative estimates of stator heat transfer.

\section{ACKNOWLEDGEMENTS}

The authors would like to thank Mr Jim Ford and Mr Gary Austin at City University for their assistance with construction of the experimental rig.

\section{REFERENCES}

[1] J. Gieras, R. Wang, and M. Kamper, Axial flux permanent magnet brushless machines, 2nd ed. Springer Verlag, 2008.

[2] P. Mellor, D. Roberts, and D. Turner, "Lumped parameter thermal model for electrical machines of TEFC design," Electric Power Applications, IEE Proceedings B, vol. 138, no. 5, pp. 205-218, 1991.

[3] S. Scowby, R. Dobson, and M. Kamper, "Thermal modelling of an axial flux permanent magnet machine," Applied Thermal Engineering, vol. 24, no. 2, pp. 193-207, 2004.

[4] R. Wang, M. Kamper, and R. Dobson, "Development of a thermofluid model for Axial field permanent-magnet Machines," Energy Conversion, IEEE Transactions on, vol. 20, no. 1, pp. 80-87, 2005.

[5] C. Lim, J. Bumby, R. Dominy, G. Ingram, K. Mahkamov, N. Brown, A. Mebarki, and M. Shanel, "2-D Lumped-parameter thermal modelling of axial flux permanent magnet generator," Proceedings of the 2008 International Conference on Electrical Machines, 2008.

[6] G. Airoldi, G. Ingram, K. Mahkamov, J. Bumby, R. Dominy, N. Brown, A. Mebarki, and M. Shanel, "Computations on heat transfer in axial flux permament magnet machines," Proceedings of the 2008 International Conference on Electrical Machines, 2008.

[7] G. Airoldi, J. Bumby, C. Dominy, G. Ingram, C. Lim, K. Mahkamov, N. Brown, A. Mebarki, and M. Shanel, "Air flow and heat transfer modeling of an axial flux permanent magnet generator," World Academy of Science, Engineering and Technology, vol. 58, pp. 809-813, 2009.

[8] D. Howey, A. Holmes, and K. Pullen, "Measurement of stator heat transfer in air-cooled axial flux permanent magnet machines," Proceedings of IECON '09, the 35th Annual Conference of the IEEE Industrial Electronics Society, pp. 1193-1198, 2009.

[9] — , "Radially resolved measurement of stator heat transfer in a rotorstator disc system," International Journal of Heat and Mass Transfer, vol. 53, no. 1-3, pp. 493-501, 2010.

[10] S. Poncet, M. Chauve, and R. Schiestel, "Batchelor versus Stewartson flow structures in a rotor-stator cavity with throughflow," Physics of Fluids, vol. 17, p. 075110, 2005

[11] J. Owen, C. Haynes, and F. Bayley, "Heat Transfer from an Air-Cooled Rotating Disk," Proceedings of the Royal Society of London. Series A Mathematical and Physical Sciences (1934-1990), vol. 336, no. 1607, pp. 453-473, 1974.

[12] R. Bunker, D. Metzger, and S. Wittig, "Local Heat Transfer in Turbine Disk Cavities: Part I, Rotor and Stator Cooling With Hub Injection of Coolant," ASME Journal of Turbomachinery, vol. 114, p. 211, 1992.

[13] Z. Yuan, N. Saniei, and X. Yan, "Turbulent heat transfer on the stationary disk in a rotor-stator system," International Journal of Heat and Mass Transfer, vol. 46, no. 12, pp. 2207-2218, 2003.

[14] H. Iacovides and J. Chew, "The computation of convective heat transfer in rotating cavities," International Journal of Heat and Fluid Flow, vol. 14, no. 2, pp. 146-154, 1993.

[15] R. Jacques, P. Le Quere, and O. Daube, "Axisymmetric numerical simulations of turbulent flow in rotor stator enclosures," International Journal of Heat and Fluid Flow, vol. 23, no. 4, pp. 381-397, 2002.

[16] F. Menter, "Two-equation eddy-viscosity turbulence models for engineering applications," AIAA Journal, vol. 32, no. 8, pp. 1598-1605, 1994.

[17] N. Gregory, J. Stuart, and W. Walker, "On the stability of threedimensional boundary layers with application to the flow due to a rotating disk," Philosophical Transactions of the Royal Society of London. Series A, Mathematical and Physical Sciences, pp. 155-199, 1955.

[18] F. Menter, R. Langtry, S. Likki, Y. Suzen, P. Huang, and S. Völker, "A Correlation-Based Transition Model Using Local VariablesPart I: Model Formulation," Journal of Turbomachinery, vol. 128, p. 413, 2006. 